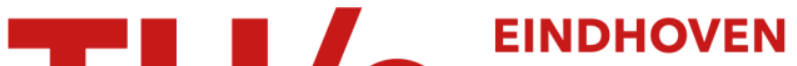 \\ UNIVERSITY OF \\ TECHNOLOGY
}

\section{Adaptive tactical pricing in multi-agent supply chain markets using economic regimes}

\section{Citation for published version (APA):}

Hogenboom, A., Ketter, W., Dalen, van, J., Kaymak, U., Collins, J., \& Gupta, A. (2015). Adaptive tactical pricing in multi-agent supply chain markets using economic regimes. Decision Sciences, 46(4), 791-818.

https://doi.org/10.1111/deci.12146

DOI:

10.1111/deci.12146

Document status and date:

Published: 01/08/2015

\section{Document Version:}

Publisher's PDF, also known as Version of Record (includes final page, issue and volume numbers)

\section{Please check the document version of this publication:}

- A submitted manuscript is the version of the article upon submission and before peer-review. There can be important differences between the submitted version and the official published version of record. People interested in the research are advised to contact the author for the final version of the publication, or visit the $\mathrm{DOI}$ to the publisher's website.

- The final author version and the galley proof are versions of the publication after peer review.

- The final published version features the final layout of the paper including the volume, issue and page numbers.

Link to publication

\section{General rights}

Copyright and moral rights for the publications made accessible in the public portal are retained by the authors and/or other copyright owners and it is a condition of accessing publications that users recognise and abide by the legal requirements associated with these rights.

- Users may download and print one copy of any publication from the public portal for the purpose of private study or research.

- You may not further distribute the material or use it for any profit-making activity or commercial gain

- You may freely distribute the URL identifying the publication in the public portal.

If the publication is distributed under the terms of Article $25 \mathrm{fa}$ of the Dutch Copyright Act, indicated by the "Taverne" license above, please follow below link for the End User Agreement:

www.tue.nl/taverne

Take down policy

If you believe that this document breaches copyright please contact us at:

openaccess@tue.nl

providing details and we will investigate your claim. 


\title{
Adaptive Tactical Pricing in Multi-Agent Supply Chain Markets Using Economic Regimes
}

\author{
Alexander Hogenboom ${ }^{\dagger}$ \\ Econometric Institute, Erasmus University Rotterdam, Rotterdam, The Netherlands, \\ e-mail: hogenboom@ese.eur.nl
}

Wolfgang Ketter and Jan van Dalen

Rotterdam School of Management, Erasmus University Rotterdam, Rotterdam, The Netherlands,e-mail:wketter@rsm.nl,jdalen@rsm.nl

Uzay Kaymak

Industrial Engineering \& Innovation Sciences, Eindhoven University of Technology, Eindhoven, The Netherlands, e-mail: u.kaymak@ieee.org

\section{John Collins}

Computer Science and Engineering, University of Minnesota, Minneapolis, MN, USA,

e-mail: jcollins@cs.umn.edu

\author{
Alok Gupta \\ Carlson School of Management, University of Minnesota, Minneapolis, MN, USA, \\ e-mail: agupta@csom.umn.edu
}

\begin{abstract}
In today's complex and dynamic supply chain markets, information systems are essential for effective supply chain management. Complex decision making processes on strategic, tactical, and operational levels require substantial timely support in order to contribute to organizations' agility. Consequently, there is a need for sophisticated dynamic product pricing mechanisms that can adapt quickly to changing market conditions and competitors' strategies. We propose a two-layered machine learning approach to compute tactical pricing decisions in real time. The first layer estimates prevailing economic conditions-economic regimes-identifying and predicting current and future market conditions. In the second layer, we train a neural network for each regime to estimate price distributions in real time using available information. The neural networks compute offer acceptance probabilities from a tactical perspective to meet desired sales quotas. We validate our approach in the trading agent competition for supply chain management. When competing against the world's leading agents, the performance of our system significantly improves compared to using only economic regimes to predict
\end{abstract}

${ }^{\dagger}$ Corresponding author 
prices. Profits increase significantly even though the prices and sales volume do not change significantly. Instead, tactical pricing results in a more efficient sales strategy by reducing both finished goods and components inventory costs.

\section{Subject Areas: Dynamic Pricing, Economic Regimes, Supply Chain Man- agement, Auctions, and Multi-agent systems.}

\section{INTRODUCTION}

In today's supply chains for durable goods, decision processes on strategic, tacti$\mathrm{cal}$, and operational levels are complicated by the complexity of logistics systems, which can encompass many interrelated entities. This increase in complexity is due to increased flexibility (such as multi-sourcing arrangements) and dynamic relationships among supply chain entities due to global market forces. Hence, effective supply chain management (SCM), focused on flexible and dynamic relationships among supply chain entities, is vital to the competitiveness of manufacturers within the supply chain. Effective SCM enables manufacturers to respond to changing market demands in a timely and cost-effective manner (Chopra \& Meindl, 2004) and can thus improve their agility. Real-time decision making technologies are becoming increasingly crucial due to the prevalence of e-business (Swaminathan \& Tayur, 2003).

A major challenge in SCM is dynamic product pricing under uncertain demand for a single product (Araman \& Caldentey, 2009; Besbes \& Zeevi, 2009) or multiple products (van Ryzin \& Vulcano, 2004; Adida \& Perakis, 2010). When flexible and dynamic relationships between supply chain entities stimulate manufacturers of durable goods to compete for customer orders, optimal product prices must account for factors other than customer demand, such as competitors' strategies or market conditions (Dasgupta \& Hashimoto, 2004; Saha, Biswas, \& Sen, 2005; Ketter, Collins, Gini, Gupta, \& Schrater, 2009, 2012; Sohn, Moon, \& Seok, 2009). This requires sophisticated dynamic product pricing, facilitated by intelligent automated decision support systems (Bichler, Gupta, \& Ketter, 2010).

We consider a dynamic supply chain environment where customer demand arrives in the form of request for quotes (RFQs) containing product specifications, due date, and reserve price. Manufacturers operate in an oligopolistic environment and competitively bid for customer orders. This market is characterized by high volatility of price and demand, as well as low market transparency. Therefore, manufacturers need to make strategic and tactical decisions about procurement, production, and pricing. Prior research (Ketter et al., 2009, 2012) has shown that economic regimes (ERs) characterize market conditions by detecting distinguishable statistical patterns in historical market data. They provide a way of intuitively and meaningfully characterizing and modeling market conditions, such as scarcity or oversupply, without a need for explicit modeling of individual aspects of the market. They provide valuable indications such as price trend and price distribution predictions over a planning horizon. The original single-layered ER model uses scarce observations of historical prices as signals for present and future price distributions, which capture the ER of the market as a latent variable. This model successfully supports product pricing, as it enables manufacturers to estimate 
present and future distributions of realized prices and adjust their strategic pricing strategies accordingly.

However, some characteristics of the single-layered ER model impede tactical applicability of this model in highly competitive settings. First, the model as proposed by Ketter et al. $(2009,2012)$ is more concerned with predictions of changes in ERs over time to make strategic decisions, such as sales quotas over a longer planning horizon. A focus on predicting short-term prices to meet these sales quotas could improve the supply chain throughput resulting in better profits, as compared to only using strategic information provided by the regimes. Second, the pricing approach using one-layered ERs uses a single pricing model. In the present study, we use different pricing models for different regimes by training a separate neural network for each regime. The individual neural networks estimate the parameters for different log-logistic distributions associated with price distributions for each regime. We update each neural network in real time by using available market information.

In this article, our overall objective is to develop a dynamic product pricing model with the ability to adapt to real-time information and to investigate whether and how such a model contributes to profit maximization. With the help of the original ER model, we partition the data into separate regime-specific training sets, capturing the relation between real-time available information and price distributions under different market circumstances. This enables us to use the original ER model in order to adapt our price distribution estimations to identified market circumstances. The proposed model captures the pricing behavior of competitors at the most detailed level and individual RFQs by individual customers. Beside observed order prices, we consider real-time available information such as RFQ characteristics, e.g., requested quantities, lead times, and reservation prices, in this process.

We validate our approach in a highly competitive, simulated setting, i.e., the trading agent competition for SCM (TAC SCM) (Collins et al., 2005; Collins, Ketter, \& Sadeh, 2010b). In this setting, we compare the performance of our MinneTAC trading agent (Collins, Ketter, \& Gini, 2009, 2010a) guided by the original ER model with the performance of MinneTAC guided by our novel two-layered ER model. The use of simulation platforms to evaluate supply network phenomena is not new. Swaminathan, Smith, and Sadeh (1998) present a modeling framework to build models of supply chains, which combines structural elements, e.g., manufacturers, retailers, or service providers, with control elements, e.g., pricing, production, or inventory policies. Van der Zee and Van der Vorst (2005) take these platforms as a communicative means, and present their framework consisting of agents and jobs, naturally embedding planning and control. In a positioning paper, Pathak, Day, Nair, Sawaya, and Kristal (2007) interpret supply networks as complex adaptive systems, thus integrating SCM research into complexity theory, while offering an approach to developing and corroborating theories about real supply networks. Nair, Narasimhan, and Choi (2009) have a specific implementation of such an approach, which models network agents as cellular automata incorporating game-theoretic principles. Their implementation aims at evaluating the development of (non-)cooperative behavior of agents in a supply network over time.

In contrast, TAC SCM, organized since 2003, aims at promoting high-quality research into the trading agents per se, as well as into the interplay between 
these agents and their supply chain environments. TAC SCM is one of the five benchmarking simulations of the trading agent community (Ketter \& Symeonidis, 2012). The Power TAC benchmarking simulation is the most recent one focusing on retail energy markets (Ketter, Collins, \& Reddy, 2013a; Ketter, Peters, \& Collins, 2013b). TAC SCM models a three-tier supply chain in which a set of manufacturers must compete with each other for parts from a set of suppliers, schedule production in their factories, manage their inventories of raw materials and finished goods, compete with each other to sell their finished goods, and ship finished goods to customers to meet their lead-time commitments. Customer demand and parts availability from suppliers are variable, finished-goods prices are set through a reverse-auction market, and supplier prices are a function of demand/supply ratios. Public information includes customer demand and the price range for each finished good type in the daily auctions; the competing manufacturing agents must interact with the markets in order to observe supplier prices and to estimate demand curves for finished goods. A typical simulation models a one-year product life cycle as 220 "days" over a period of about 1 hour. Further information regarding the different competitions and tournament schedules can be found on the Association for Trading Agents Research Web site (http://www.tradingagents.org).

The contribution of this article is a novel two-layered machine learning model for tactical sales management in supply chain markets for durable goods with limited information and high uncertainty. It achieves this by focusing on predicting economic conditions using the original ERs model (first layer), estimating price distributions for each regime using fast neural networks (second layer), and using these price distributions to develop efficient sales strategies.

The article is organized as follows. First, we discuss related work on dynamic product pricing. Our own product pricing approach is introduced in the subsequent two sections. Then, we evaluate the novel approach. In the last section, we conclude and provide directions for future research.

\section{RELATED WORK}

Previous research has revealed a number of important aspects of dynamic product pricing: the role of acceptance probabilities, the need for market responsive pricing policies, and the dependence of different market conditions. Acceptance probabilities indicate the likelihood that customers actually accept an offer and proceed to buy the product at the offered price. Estimated acceptance probabilities are useful for sellers in developing pricing policies to meet sales targets. Recent research supports the viability of this approach. Dasgupta and Hashimoto (2004), for instance, model product prices using a dynamic pricing algorithm based on an estimated distribution of buyer reservation prices. Reversing the cumulative reservation price distribution gives the proportion of customers willing to buy the product as a function of the offered price. This function can subsequently be used to determine the price that is expected to yield a specified sales quota. Likewise, Walsh, Parkes, Sandholm, and Boutilier (2008) use estimated distributions of buyer's private values to model acceptance probabilities. Other ways of modeling acceptance probabilities include linear regression of acceptance rates on offer prices (Pardoe \& Stone, 2006b), offline-trained acceptance probability 
distributions, and modeling the decision function of the accepting entities, based on decision histories, e.g., using Chebychev polynomials (Saha et al., 2005).

In addition, dynamic product pricing should be responsive to market situations. Tang and Yin (2007), for instance, show for a situation with known demand and uncertain supply that a responsive pricing policy, in which the retail price is determined after observing the realized supply, results in a higher expected profit than a pricing policy that discards realized supply. Consequently, modeling expected or observed supply-side behavior in the product pricing process contributes to profit maximization. Furthermore, Kovalchuk and Fasli (2008) and Li, Giampapa, and Sycara (2004) consider current and future offers of competitors, defined as outside options. Sohn et al. (2009) analyze such outside options by quantifying a product's own price change together with the relative prices of competing products in a price elasticity. Optimal pricing policies are then selected based on scenario analysis for various outcomes of this price elasticity. Groves, Collins, Gini, and Ketter (2014) propose an extensive set of business key performance indicators (KPIs) and apply them to analyze market dynamics and associated pricing policies in supply chains.

\section{MANAGERIAL IMPLICATIONS}

Dynamic pricing policies may benefit from information about systematically changing market conditions, known as ERs (Hathaway \& Bezdek, 1993; Mount, Ning, \& Cai, 2006). The ability of decision makers to correctly identify the current regime and predict the onset of new regimes is crucial in order to prevent over- or under-reaction to market conditions (Massey \& Wu, 2005). Different ERs, such as situations of excess demand or excess supply, may involve different optimal pricing policies.

Figure 1 demonstrates how we can use ERs to guide both tactical and strategic decision making. An example of tactical decisions would be product pricing, whereas the determination of product mix and production planning are examples of strategic sales decisions (Ketter et al., 2009, 2012). According to the classical framework of Sprague and Carlson (1982), a (real-world) decision support system typically consists of a knowledge management system (KMS), a model management system (MMS), a database management system (DMS), and a user interface system (UIS). Each of these logical components is used to monitor and regulate the flow of crucial information in order to support decision making in an organization. Figure 1 depicts the flow of both strategic and tactical information, based upon the past, present, and future state of ERs that characterize the market circumstances for products.

The DMS and the UIS in Figure 1 represent the real-world side of the system, which mainly facilitates the integration of human judgment and signals received from the environment. The information flow from supplier databases through the DMS to UIS ultimately results in visual aids on a firm's strategic monitoring dashboards, enabling operations managers to detect changing environmental conditions and to recommend remedies in anticipation of the evolving competitive environment. Most strategic decisions involving resource allocation require such human intervention. 
Figure 1: The incorporation of our regime model in real-life decision support systems, as modeled by Sprague and Carlson (1982). This visualization has been adapted from Jelinek and Bergey (2013). Solid arrows represent strategic decisions, whereas dashed arrows represent tactical decision making. Regime identification is used as a tool for tactical decision making, e.g., sales pricing. Regime prediction is used a tool for strategic decision making, i.e., resource allocation.

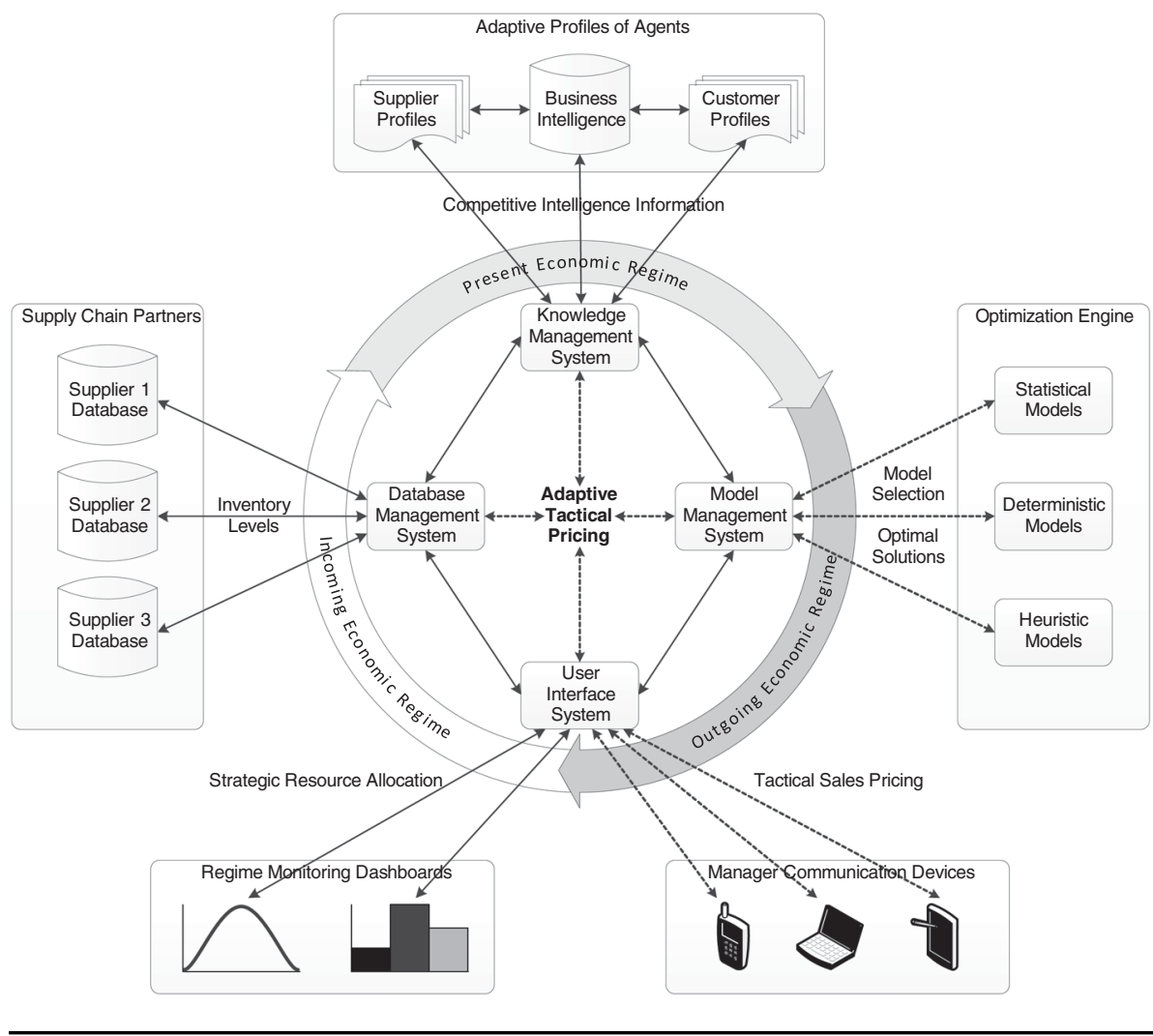

The virtual agent side of the system in the real world is represented by the KMS and MMS, which can be used to adapt tactical decisions involving product pricing to profiles of suppliers and customers in order to maximize their probability of accepting an offer. This is demonstrated by the information flow from the up-todate agent profiles in the KMS through the MMS - where the optimal price point is determined - to the UIS that provides sales managers in the field with real-time information. The KMS is used to maintain and update competitive intelligence information that is captured in the form of supplier-specific and customer-specific information, and stored as model coefficients that have been optimized from past ERs. The MMS, in turn, maintains and selects models that are appropriate for the current ER and as such delivers optimal pricing guidance.

Guided by identified past, present, and future ERs, the flow of strategic and tactical information enables economic efficiency to be derived from the system, by delivering value to the firm which would otherwise be left on the table in the 
form of economic surplus. As such, our system is an evolving adaptive platform, uniquely suited to changing environmental conditions in a practical setting. Viable product pricing strategies in dynamic markets would ideally involve the estimation of the probability that customers accept an offered price, the use of expected or observed supplier behavior and competitors' pricing, and the identification of specific, possibly changing ERs. However, a dynamic product pricing approach that takes these findings jointly into account, and is thus able to adapt to ever-changing market circumstances such as those in highly competitive complex supply chains, is yet to be proposed. In our current endeavors, we aim to take a step in this direction.

\section{PRODUCT PRICING BASED ON OFFER PRICE DISTRIBUTIONS}

Manufacturers of durable goods typically sell products in markets with differentiated customer demand. Knowledge about competitors' procurement, production, and pricing decisions is generally not available. Such knowledge can at most indirectly be inferred from unexpected increases or decreases of the volume or profitability of one's own sales. Customers may be expected to purchase the lowest priced product from any offer of equally preferred product specifications. In the real world, the customer will not always select the bid with the lowest price, for a variety of reasons that include reputation effects. In TAC SCM, there is a reputation effect with respect to suppliers, but not with respect to customers. Aggregate demand will vary over time with respect to volume and quality. Accurate predictions of the price at which customers are willing to accept offered products are essential to maintain sustainable market positions.

In this section, we model the probability that offers are accepted at a certain offer price. The model is based on the idea that past information about product sales and prices can be used to infer the distribution of offered prices in the market. Real-time application of this model requires flexible updating of the parameters involved, for which we propose artificial neural networks (ANNs), as we explain below. A summary of the mathematical notation used in this article can be found in the Appendix (Table A1).

\section{Modeling Acceptance Probabilities}

We model customer offer acceptance probabilities based on the distribution of all offered prices. In this way, we directly consider the unobservable decision making processes of all traders and obtain a complete estimation of customer acceptance probabilities. By contrast, linear regression on recent offer prices, as proposed by Pardoe and Stone (2006b) may be less effective because their regression model typically only captures price movements in one direction. Furthermore, conflating actual offer price distributions with predicted price distributions should yield a more robust approach than using only predicted individual offer prices from a model (Kovalchuk \& Fasli, 2008), where point estimates are potentially non-occurring. Using the offer price distribution for a particular RFQ, we derive order price distributions as the distribution of the order statistic of the minimum offer price. The cumulative density of order prices summarizes the probabilities that order prices are at or 
below certain values. The reverse cumulative density approximates the probability for an agent to offer better deals than other competitors and thus to obtain orders.

Let us consider a single RFQ $r$ on a particular day $d$ for a particular good $g$. Let the prices $p_{d g r}$ offered by traders in response to this RFQ be distributed in accordance with a marginal distribution $f\left(p_{d g r} ; \theta\right)$ and a cumulative distribution $F\left(p_{d g r} ; \theta\right)$, with $0<p_{d g r}<u$, i.e., prices are non-negative and have a reservation price $u$ as upper bound, and $\theta$ a vector of unknown parameters.

Assuming that customers only consider bids at or below their reservation price and always select the bids with the lowest offer price, order prices can be interpreted as realizations of the first-order statistic for the minimum of a sample of offer prices. Considering $n_{d g r}$ offer prices per RFQ, the cumulative density of the minimum offer price for that particular RFQ is the statistical order distribution (Kapadia, Chan, \& Moye, 2005):

$$
F_{1}\left(p_{d g r} ; \theta\right)=1-\left(1-F\left(p_{d g r} ; \theta\right)\right)^{n_{d g r}}, \quad 0<p_{d g r}<u, \quad n_{d g r}>0 .
$$

This cumulative distribution of order prices yields the fraction of order prices realized at or below offer price $p_{d g r}$. Hence, a trader placing an offer of $p_{d g r}^{\prime}$, incurs an $F_{1}\left(p_{d g r}^{\prime} ; \theta\right)$ risk of not acquiring the order. The probability $P\left(o \mid p_{d g r}\right)$ that a customer will place an order $o$ with a trader at an offer price $p_{d g r}$ can therefore be defined as

$$
P\left(o \mid p_{d g r}\right)=1-F_{1}\left(p_{d g r} ; \theta\right), \quad 0<p_{d g r}<u .
$$

The customer offer acceptance probability (2) holds for a single RFQ. Suppose that $m_{d g}$ RFQs are presented to the market on day $d$ for good $g$. Let $n_{d g}$ randomly sampled valid offer prices $p_{d g}$ for each out of $m_{d g}$ RFQs be identically and independently distributed in accordance with a distribution $f\left(p_{d g} ; \theta\right)$ and a cumulative distribution $F\left(p_{d g} ; \theta\right)$, with $0<p_{d g}<u$ and $\theta$ a vector of unknown parameters. For such a distribution, the cumulative distribution of the order prices over all $m_{d g}$ RFQs can be derived as

$$
F_{1}\left(p_{d g} ; \theta\right)=\left(1-\left(1-F\left(p_{d g} ; \theta\right)\right)^{n_{d g}}\right)^{m_{d g}} \quad 0<p_{d g}<u, \quad n_{d g}, m_{d g}>0,
$$

yielding an offer acceptance probability defined as

$$
P\left(o \mid p_{d g}\right)=1-\left(1-\left(1-F\left(p_{d g} ; \theta\right)\right)^{n_{d g}}\right)^{m_{d g}}, \quad 0<p_{d g}<u, \quad n_{d g}, m_{d g}>0 .
$$

Equation (4) can be used to estimate the share of received orders with respect to the total number of RFQs for product $g$ on day $d$, generated by a price offered on these RFQs. When pricing products, one may have a specific target share or sales quota. Now, let $q_{d g}^{*}$ be a ratio in the interval $[0,1]$, representing the sales quota for product $g$ on day $d$, with $m_{d g}$ associated RFQs, for each of which $n_{d g}$ prices are offered. The associated offer price $p_{d g}^{*}$ is obtained by taking the inverse probability function at $q_{d g}^{*}$. Solving the equation to $p_{d g}^{*}$ yields the optimal offer price $p_{d g}^{*}$ associated with the desired quota. Following this approach, tactical pricing decisions for day $d$ are directly linked to price distributions for day $d$ through modeled acceptance probabilities.

Utilization of the price distributions in the bidding process requires insight into $\theta$ and $n_{d g}$ in (4). When all data are available, $m_{d g}$ and $n_{d g}$ can be determined by a counting process and $\theta$ can be estimated by maximum likelihood as follows. 
When all prices in the sample of offers $p_{d g}$ for all $m_{d g}$ RFQs issued for product $g$ on day $d$ are identically and independently distributed in accordance with the offer price distribution $f\left(p_{d g r i} ; \theta\right)$, the joint distribution of all valid offer prices equals

$$
f\left(p_{d g} ; \theta\right)=\prod_{r=1}^{m_{d g}} \prod_{i=1}^{n_{d g}} f\left(p_{d g r i} ; \theta\right), \quad 0<p_{d g r i}<u,
$$

where $p_{d g r i}$ is the $i$ th of $n_{d g}$ prices offered on day $d$ for RFQ $r$ for product $g$. Parameters $\theta$ can be estimated by minimizing their negative log-likelihood function for observed offer prices $p_{d g}$, e.g., using the Newton-Raphson method (Coleman \& Li, 1996):

$$
L\left(\theta ; p_{d g}\right)=-\sum_{r=1}^{m_{d g}} \sum_{i=1}^{n_{d g}} \ln \left(f\left(p_{d g r i} ; \theta\right)\right), \quad 0<p_{d g r i}<u .
$$

The optimization of (6) is straightforward when all relevant price information is available. In real time, however, offer prices from competitors may not be available for estimating the joint distribution of offer prices (5). One solution to dealing with this problem might be to use a general probability density estimator for finding a mapping between observable quantities and the target distributions. Examples are kernel density estimation (Jones, 1989), probabilistic fuzzy systems (van den Berg, Kaymak, \& van den Bergh, 2004) and ANNs (Marchette \& Priebe, 1990). However, identifying this mapping may be hampered by the large number of parameters to estimate. Furthermore, the convenient multiplicative form of (5) would not hold in general. A more viable alternative is to select a general parametric distribution function and to use a general function approximator to determine the parameter values of the target distribution from the information available real time. In the next section, we further elaborate on this idea.

\section{Real-Time Parameter Estimation}

Real-time estimation of the distribution parameters $\theta$ and $n_{d g}$ requires the specification of a relation between these parameters and a vector of real-time available information, $\vec{x}$, possibly including information on RFQs or one's own sales. Approximations of these relations, $\hat{h}_{\theta}(\vec{x})$ and $\hat{h}_{n_{d g}}(\vec{x})$, can be found, for instance, by linear regression (Pardoe \& Stone, 2006b). However, the functional specification of the relation between the parameters and real-time available information may not always be known a priori. We therefore model these relations with ANNs, which have been proven to be useful for economic forecasts in various domains (Kovalchuk \& Fasli, 2008).

An ANN is a mathematical model inspired by biological neural networks. It provides a general, practical method for learning real-valued, discrete-valued, and vector-valued functions over continuous and discrete-valued attributes from samples in order to facilitate regression or classification (Mitchell, 1997). ANNs bring the attractive feature of fast evaluation of these functions, which is essential for real-time product pricing. Other advantages include robustness to noise in the training data (Mitchell, 1997) and support for adaptivity by adjusting the weights of each node's inputs on-the-fly using newly obtained samples. 
A drawback of standard feed-forward back-propagation ANNs is the time needed for design and training. An alternative could be a probabilistic neural network (PNN) or a radial basis function network (RBFN). These two types of ANNs are essentially quite similar, with their main difference lying in the fact that PNNs model each data point in the training set with a neuron, whereas RBFNs typically need a number of neurons that is significantly smaller than the number of data points in the training set. This gives RBFNs an attractive advantage over PNNs for our purpose, with our data typically consisting of a vast amount of data points.

In this light, we use an RBFN, a specific type of ANN that can be designed and trained in a fraction of the time it takes to design and train standard feedforward back-propagation ANNs (Mitchell, 1997). An RBFN is a two-layer ANN consisting of a hidden layer and an output layer. The activation function in each hidden unit $h$ is a local function $K_{h}\left(d\left(\vec{x}_{h}, \vec{x}\right)\right)$, where $\vec{x}$ is a vector of features, $\vec{x}_{h}$ is called the center of the local function, and $d\left(\vec{x}_{h}, \vec{x}\right)$ is the (Euclidean) distance between $\vec{x}_{h}$ and $\vec{x}$. $K_{h}(z)$ is a so-called kernel function whose value is at a maximum when $z=0$, and approaches zero for large values of $z$. The local functions in the hidden layer are typically Gaussians (Mitchell, 1997), centered at $\vec{x}_{h}$ with variance $\sigma_{h}^{2}$. The number of Gaussians $H$ is subject to optimization and their centers can be determined by clustering the data in various ways, for example, using the $k$ means algorithm (MacQueen, 1967). The variance $\sigma_{h}^{2}$ of each Gaussian may, for example, be estimated as the largest squared distance between the centers. The network's output for an instance $\vec{x}, \hat{h}(\vec{x})$, is a linear combination of the activation units, weighted for their weights $w_{h}$, and a bias $w_{0}$ (Mitchell, 1997):

$$
\begin{gathered}
\hat{h}(\vec{x})=w_{0}+\sum_{h=1}^{H} w_{h} K_{h}\left(d\left(x_{h}, \vec{x}\right)\right), \\
K_{h}\left(d\left(x_{h}, \vec{x}\right)\right)=e^{\frac{1}{2 \sigma_{h}^{2}} d^{2}\left(x_{h}, \vec{x}\right)} .
\end{gathered}
$$

An RBFN is a global approximation $\hat{h}(\vec{x})$ of a target function $h(\vec{x})$, represented as a linear combination of local functions around their centers. It has a relatively easy design and training stage, it is robust, has the possibility to introduce adaptivity, and has fast real-time evaluation capabilities. By using an RBFN to map real-time available information to distribution parameter values, our product pricing model is responsive to observable information in a predefined way. However, this mapping as such is essentially static and should be updated at runtime in order to adapt to changing market conditions and market responses. Therefore, we introduce adaptivity to the model by using ERs (Ketter et al., 2009, 2012).

\section{ADAPTIVE PRODUCT PRICING USING ECONOMIC REGIMES}

Adaptive, real-time product pricing requires dynamic modeling of the relations between available data and price distributions based on market conditions. Additionally, we incorporate market feedback by means of error terms that reflect the market's response to our pricing decisions.

In real-world scenarios, market conditions offer only limited visibility. Previous research (Ketter et al., 2009, 2012) identified distinct market conditions through economic principles and machine learning techniques. Historical data were used 
to compute price distributions that, in turn, enabled the estimation of sales prices and the identification of ERs (e.g., scarcity, a balanced situation, or oversupply). Here, we add real-time adaptivity using observable market information.

Realized order prices and order probabilities tend to vary with the distribution of ERs (Ketter et al., 2009, 2012), which affects the relations between available data and price distributions. Hence, we differentiate product pricing strategies with respect to ERs. Suppose that market conditions can be meaningfully summarized by means of $M$ regimes. None of these regimes can be known to occur with certainty. Instead, we assume that each day $d$, each of these regimes, $R_{d g k}$, occurs with probability $P\left(R_{d g k}\right)$ for product $g$, as calculated by applying the regime model proposed by Ketter et al. $(2009,2012)$. The regime with the highest probability of occurrence is labeled as dominant regime on day $d$. For each regime $k=1, \ldots, M$, price distribution parameters $\theta_{k}$ and $n_{d g k}$ (and $m_{d g}$, in case of predictions) for product $g$ on day $d$ are estimated using RBFNs. Based on these $M$ estimated distributions, acceptance probabilities $P\left(o_{k} \mid p_{d g k}\right)$ are derived, which are subsequently weighted with the associated regime probabilities $P\left(R_{d g k}\right)$ for regime $R_{d g k}$, to have the resulting acceptance probability estimations reflect the market conditions.

Updating the weights in the RBFNs based on real-time information is often not feasible due to limited visibility of, for instance, the realized sales price distributions. Instead, daily estimated acceptance probabilities may be adjusted directly by multiplying the probabilities with a correction factor $\epsilon$ defined as the ratio of actually received orders and predicted orders, as proposed by Pardoe and Stone (2006a) and Ketter et al. (2012). This ratio feeds market responses back to the model in real time. Using performance information up until day $d-1$, the error term can be smoothed, yielding $\widetilde{\epsilon}_{(d-1) g k}$ for each regime $k$, to prevent over- or under-compensation.

Obviously, acceptance probabilities $P\left(o_{k} \mid p_{d g k}\right)$ range from 0 to 1 . But if we straightforwardly adopt the suggested correction, adjusted acceptance probabilities $P\left(o_{k} \mid p_{d g k}\right)^{\prime}$ will be in the range $\left[0, \widetilde{\epsilon}_{(d-1) g k}\right]$. This implies that for $q_{d g}^{*} \geq \widetilde{\epsilon}_{(d-1) g k}$, no suitable price can be found, which is clearly undesirable in case $\widetilde{\epsilon}_{(d-1) g k}<$ 1. Therefore, we introduce the feedback correction as an exponent of the offer acceptance probability as follows:

$$
\begin{aligned}
P\left(o_{k} \mid p_{d g k}\right)^{\prime}= & P\left(o_{k} \mid p_{d g k}\right)^{\tilde{\epsilon}_{(d-1) g k}}, \quad 0<p_{d g k}<u, \quad \widetilde{\epsilon}_{(d-1) g k}>0, \\
= & \left(1-\left(1-\left(1-F\left(p_{d g k} ; \theta_{k}\right)\right)^{n_{d g k}}\right)^{m_{d g}}\right)^{\tilde{\epsilon}_{(d-1) g k}}, \\
& 0<p_{d g k}<u, \quad n_{d g k}, m_{d g}, \widetilde{\epsilon}_{(d-1) g k}>0,
\end{aligned}
$$

The adjusted acceptance probabilities range from 0 to 1 for $0<p_{d g k}<u$ after correction for the smoothed correction term $\widetilde{\epsilon}_{(d-1) g k}$. For each regime $k$, expression (9) is used to determine the optimal corrected offer price $p_{d g}^{*^{\prime}}$ corresponding with the desired sales quota $q_{d g}^{*}$ for product $g$ on day $d$ by solving the equation $P\left(o_{k} \mid p_{d g k}^{*^{\prime}}\right)^{\prime}=q_{d g}^{*}$ to $p_{d g k}^{*^{\prime}}$. An overall corrected offer price $p_{d g}^{*^{\prime}}$ can then be obtained by averaging the regime-based prices using the associated regime probabilities $P\left(R_{d g k}\right)$ as weights. 
The correction term considered in (9) should be assigned values such that under each dominant regime $k$, the expected customer offer acceptance probabilities $P\left(o_{k} \mid p_{(d-1) g}^{*^{\prime}}\right)$ associated with offer price $p_{(d-1) g}^{*^{\prime}}$ are corrected by the unsmoothed correction terms $\epsilon_{(d-1) g k}$ to the proportion of actually received number of orders $q_{(d-1) g}$. The correction terms can subsequently be smoothed. Hence, offer price and customer response should be related as follows:

$q_{(d-1) g}=P\left(o_{k} \mid p_{(d-1) g}^{*^{\prime}}\right)^{\epsilon_{(d-1) g k}}, \quad 0<q_{(d-1) g}<1, \quad 0<p_{(d-1) g}^{*^{\prime}}<u, \quad \epsilon_{(d-1) g k}>0$.

In an attempt to track the trend in the correction terms and thus to provide an accurate estimate of the error of the model on day $d$, given information up until day $d-1$, the correction terms can be smoothed using exponential smoothing. Here, the smoothing factor $\beta$ is taken to be proportional to the associated regime probabilities in order to attribute errors only to the models responsible for these errors. Smoothing is done by linearly combining the latest correction term (11) and the previous smoothed correction:

$$
\begin{gathered}
\epsilon_{(d-1) g k}=\frac{\ln \left(q_{(d-1) g}\right)}{\ln \left(P\left(o_{k} \mid p_{(d-1) g}^{*^{\prime}}\right)\right)}, \quad 0<q_{(d-1) g}<1, \quad 0<P\left(o_{k} \mid p_{(d-1) g}^{*^{\prime}}\right)<1, \\
\tilde{\epsilon}_{(d-1) g k}=\beta P\left(R_{(d-1) g k}\right) \epsilon_{(d-1) g k}+\left(1-\beta P\left(R_{(d-1) g k}\right)\right) \tilde{\epsilon}_{(d-2) g k} .
\end{gathered}
$$

Summing up, the model assumes some distribution to be underlying offer prices, the parameters of which can be estimated real time based on available data using RBFNs. The relation between the available data and the parameters is dynamically modeled using ERs (characterizing market conditions) and error terms (accounting for customer feedback), thus enabling adaptive product pricing. For now, predicted effects of the resulting dynamic pricing behavior on market conditions are not directly taken into account when pricing products using the proposed model, yet the model can always adapt to newly instigated market conditions immediately after the fact. The online evaluation of available data by means of the RBFNs is a matter of milliseconds and has a computational complexity of $O(G M)$, with $G$ denoting the number of distinct products and $M$ representing the number of regimes.

\section{IMPLEMENTATION IN A SOFTWARE AGENT}

The model as proposed in the previous sections assumes a general offer price distribution, which is yet to be specified. We propose to use a log-logistic distribution, as this distribution covers a variety of shapes depending on the parameters $\alpha$ and $\gamma$ and has the attractive feature that an analytical closed-form expression exists for the cumulative density function. Moreover, according to the Kolmogorov-Smirnov test with a significance level of 0.05 , this distribution appears to adequately describe the data in TAC SCM in over $60 \%$ of the analyzed price samples from historical data, i.e., TAC SCM 2007 Semi-Finals and Finals (9323-9327tac5 and 7308-7312tac3) (Swedish Institute for Computer Science, 2004-2012) and TAC 
SCM 2008 Semi-Finals and Finals (763-768tac02 and 794-799tac01) (University of Minnesota, 2003-2012) game data. The log-logistic distribution $f(p ; \alpha, \gamma)$ and its cumulative form $F(p ; \alpha, \gamma)$ (Mood, Graybill, \& Boes, 1974) are truncated such that the distribution is defined on the domain $0<p<u$ and parameterized such that $\alpha$ represents the median and $\gamma$ quantifies the distribution tightness:

$$
\begin{gathered}
f(p ; \alpha, \gamma)=\frac{\left(\alpha^{-\gamma}-u^{-\gamma}\right) \gamma p^{-\gamma-1}}{\left(\alpha^{-\gamma}-2 u^{-\gamma}+p^{-\gamma}\right)^{2}}, \quad 0<p<u, \quad \alpha, \gamma>0, \\
F(p ; \alpha, \gamma)=\frac{\alpha^{-\gamma}-u^{-\gamma}}{\alpha^{-\gamma}-2 u^{-\gamma}+p^{-\gamma}}, \quad 0<p<u, \quad \alpha, \gamma>0 .
\end{gathered}
$$

When $F\left(p_{d g k} ; \theta_{k}\right)$ in (9) is substituted for (14), the corrected price $p_{d g}^{*^{\prime}}$ expected to yield a required sales quota, i.e., acceptance probability (Ketter et al., 2012), $q_{d g}^{*}$ can be obtained as

$$
\begin{gathered}
q_{d g}^{*^{\prime}}=\sqrt[n_{d g k}]{1-\sqrt[m_{d g}]{1-\tilde{\epsilon}_{(d-1)} \sqrt[g k]{q_{d g}^{*}}}}, \quad 0<q_{d g}^{*}<1, \\
m_{d g}, n_{d g k}, \tilde{\epsilon}_{(d-1) g}>0, \\
p_{d g}^{*^{\prime}}=\left(\frac{u^{-\gamma_{k}}\left(\alpha_{k}^{-\gamma_{k}}\left(u^{\gamma_{k}}-2 \alpha_{k}^{\gamma_{k}}\right) q_{d g}^{*^{\prime}}+1\right)}{1-q_{d g}^{*^{\prime}}}\right)^{-\frac{1}{\gamma_{k}}}, \quad \sum_{k=1}^{M} P\left(R_{d g k}\right) p_{d g k}^{*^{\prime}}, \quad 0<p_{d g k}^{*^{\prime}}<u,
\end{gathered}
$$

with $u=1.25$ (as normalized prices in TAC SCM are never above 1.25 of nominal cost) and the number of considered regimes $M=5$, as extensively discussed by Ketter et al. $(2009,2012)$. In TAC SCM, the number of offers per RFQ $n_{d g}$ is approximated by the mean number of offers per RFQ for product $g$ on day $d$, which is bounded by the number of participating agents.

Dynamic product pricing in TAC SCM can now be done using (15)-(17). Due to limited visibility of market conditions, the $\alpha_{k}, \gamma_{k}$, and $n_{d g k}$ parameters for product $g$ on day $d$ for dominant regime $k$ are to be estimated using RBFNs. Further adaptivity of the framework can be realized by utilizing (11) and (12) to account for customer feedback. We dynamically scale the learning rate $\beta$ to range between 0.15 and 0.85 (as determined using a hill-climbing procedure), depending on the distance of the model's median with respect to the price-following median of observed minimum and maximum prices.

\section{Implementation in the MinneTAC Agent}

The sales decisions made by the MinneTAC agent (Collins et al., 2009, 2010a) originate from price predictions based on market observations, and are characterized separately for each of the three market segments. These ERs have been characterized as extreme scarcity, scarcity, a balanced situation, oversupply, and extreme oversupply (Ketter et al., 2009, 2012). On day $d$, the (dominant) regime for good $g$ is identified as the regime having the highest likelihood of occurrence, 
given the estimated normalized mean price for that day. We estimate the normalized mid-range price, $\widetilde{\mathrm{np}}_{d g}$, as the average of the double exponentially smoothed normalized minimum and maximum price observed in the market. The normalized price $\mathrm{np}_{d g}$ for good $g$ on day $d$ is the ratio of actual price to nominal production cost, which is the sum of the nominal costs of the parts required to build a product. Normalized prices rarely fall below half of nominal cost, since this is the lowest cost available from suppliers, and never rise above 1.25 of nominal cost, the upper bound on customer reservation prices.

A price density function has been modeled on historical normalized order price data by using a Gaussian mixture model (GMM) (Titterington, Smith, \& Makov, 1985) with 25 Gaussians, whose number reflects a balance between prediction accuracy and computational overhead. Clustering these price distributions over time periods, using the $k$-means algorithm, yields distinguishable statistical patterns that can be interpreted as ERs (Ketter et al., 2009, 2012). In TAC SCM, price information is available up until the preceding day. Hence, the MinneTAC agent estimates the mean price of the current day $d$ using an exponentially smoothed prediction of $\widetilde{n p}_{d g}$, and then returns the regime probabilities for day $d$. When this estimate is supplied to the model, each individual Gaussian in the model is activated to its own distinct extent, thus generating an expected price distribution. Subsequently normalizing all clusters' price densities enables determination of regime probabilities. Price distributions and regime probabilities for future days are determined by using Markov prediction and Markov correction-prediction processes for short-term and long-term decision making, respectively (Ketter et al., 2012). After determining current and future regime probabilities, products are priced using offer acceptance probabilities, such that sales quotas are fulfilled.

In our benchmark configuration, the sales process is fully driven by the ER model (Ketter et al., 2009, 2012). Median product prices and future price trends are derived from order price distributions estimated by this model. The trends and medians, along with cost estimates and resource constraints, are inputs to a linear program that computes sales quotas to maximize profits over a medium-term time horizon. Given these daily sales quotas, optimal offer prices are computed as the price that will sell a quota against a probability that is specified by an order acceptance probability curve. This curve is approximated using the order price distributions estimated by the ER model. In order to compensate for uncertainty in generated predictions, interval randomization is applied to offer prices, which adds a slight variability to these prices. The ER model underlying the sales process in this benchmark configuration does not adapt to real-time available information or customer feedback.

The price distributions estimated by the ER model are updated online based on observed minimum and maximum prices, but they do not account for other factors like RFQ characteristics and lack full adaptivity. Previous research has shown the effectiveness of ERs on predominantly a strategic level of decision making (Ketter et al., 2009, 2012), yet the lack of adaptivity of the model renders it less useful for decision making on a tactical level, such as product pricing. In an attempt to improve the product pricing process by combining regime information with real-time available data, we replace the ER model with a dynamic ER (DynER) 
Figure 2: Schematic overview of the DynER sales process for an arbitrary product.

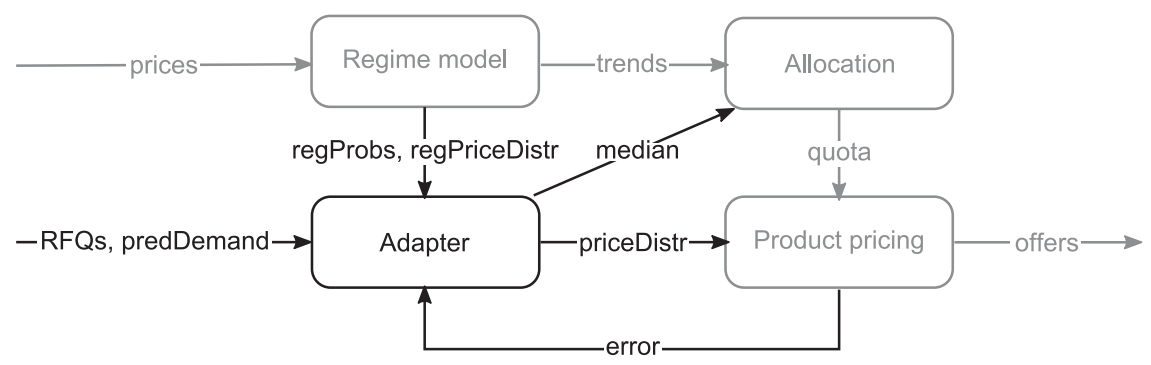

model. We implement the proposed adaptivity for daily product pricing decisions on a tactical level. The algorithm (Algorithm 1) involves parameter estimation using RBFNs and subsequent product pricing based on (15) through (17). A correction term is used, following (11) through (12).

In order to introduce adaptivity into the ER model, we built an adapter for the outputs of the ER model (see Figure 2). The adapter uses RBFNs that have been trained offline to transform order price distributions estimated by the ER model into parameterized order price distributions per dominant regime and assigns weights to these distributions, equal to their associated regime probabilities. These adaptations are based on RFQ characteristics and predicted demand. The adapted distributions can then be used for product pricing. A parameterized acceptance probability function can be derived per dominant regime. Given a quota for a product (specified by the agent's allocation component), the product pricing component uses the adapter to compute the price that is expected to yield this quota per dominant regime and weights the suggested prices with their associated regime probabilities. Then, offers are made on all selected RFQs for the considered product, in an interval around the optimized price. The market responses to these offers are fed back to the adapter.

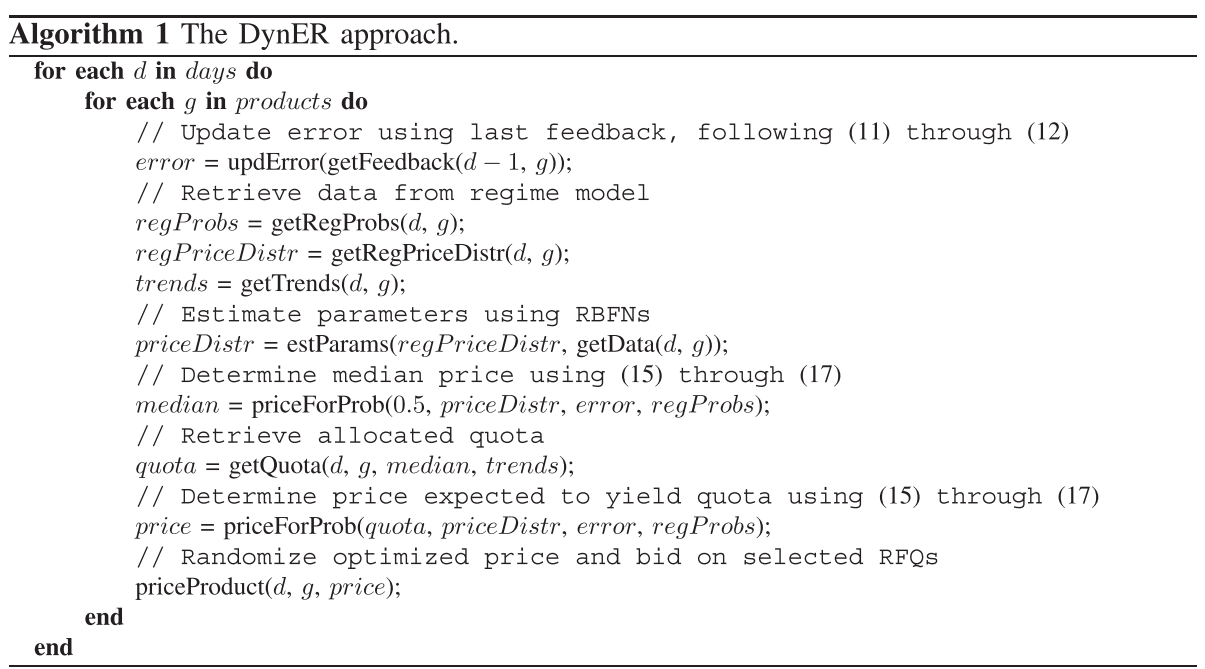




\section{Radial Basis Function Network Training}

In order for the DynER approach to work in the MinneTAC agent for TAC SCM, we need to train RBFNs (offline) for real-time estimation of unobservable price distribution parameters. To this end, we utilize TAC SCM 2007 Semi-Finals and Finals (9321-9328tac5 and 7306-7313tac3) (Swedish Institute for Computer Science, 2004-2012) and TAC SCM 2008 Semi-Finals and Finals (761-769tac02 and 792-800tac01) (University of Minnesota, 2003-2012) game data. The first two games and the last game per server form the test set, and all other games form the training set. For each dominant regime $k$, an RBFN is trained to estimate $\alpha_{k}, \gamma_{k}$, and $n_{d g k}$ for product $g$ on day $d$. Training and test datasets are split into datasets per dominant regime, identified by the current ER model.

We adapt regime-based price distributions done using the GMM implemented in the MinneTAC agent in order for them to be useful in the daily product pricing process. Hence, these regime-based price distributions are used as RBFN inputs. Other RBFN inputs include the product type, the day, the RFQ characteristics of the product for that day, and observable historical prices. We characterize the regime-based price distributions by their 10th, 50th, and 90th percentiles. The RFQ characteristics of the product for that day include the number of RFQs, the mean and standard deviation of their reservation prices, the mean and standard deviation of the requested quantities, and the mean and standard deviation of the lead times. The observable historical prices are characterized by the minimum, maximum, and mid-range prices of the preceding day, both nominal and exponentially smoothed.

When adapting regime-based price distributions with our RBFNs, we use data on product type and day because of the heterogeneity of products and the volatility of their associated prices in TAC SCM. RFQ characteristics are incorporated, since competitors' pricing strategies are likely to depend on these characteristics. Reservation prices, for example, give an indication of the maximum price manufacturers will bid. Also, the number of simultaneous similar bidding processes affects the associated revenues due to their (partial) substitutivity (Walsh et al., 2008). Observable (historical) prices can also provide more insight into competitors' pricing strategies (Kovalchuk \& Fasli, 2008; Ketter et al., 2009, 2012).

Using our historical TAC SCM data, target parameter values can be determined by counting (for $n_{d g k}$ ) and by fitting distributions using (6) and (13) (for $\alpha_{k}$ and $\gamma_{k}$ ). A typical training dataset thus generated contains over 15,000 observations, a typical test dataset over 8,000. The performance of RBFNs trained on the training set can be evaluated on the test set, which is sufficiently large and representative (Mitchell, 1997). For the resulting optimal values for $\gamma_{k}$, the increment in $\gamma_{k}$ needed to tighten the distribution increases as the distribution gets tighter. As the required accuracy decreases for an increasing $\gamma_{k}$, the networks are trained to predict the natural logarithm of $\gamma_{k}$.

For each regime-specific RBFN, the architecture is optimized keeping the numbers of inputs and outputs fixed. The number of hidden nodes is determined using a hill-climbing procedure, which minimizes the root mean squared deviation (RMSD) of the outputs on the test set. The RMSD equals 
Table 1: Optimized number of clusters $H$ for parameter estimating RBFNs and the RMSD of predicted parameter values from their target values per dominant regime $k$.

\begin{tabular}{ccccc}
\hline$k$ & $H$ & RMSD $\alpha_{k}$ & RMSD $\ln \left(\gamma_{k}\right)$ & RMSD $n_{d g k}$ \\
\hline 1 & 15 & 0.045 & 0.769 & 0.998 \\
2 & 15 & 0.035 & 0.698 & 1.073 \\
3 & 19 & 0.037 & 0.651 & 1.015 \\
4 & 30 & 0.039 & 0.641 & 0.944 \\
5 & 23 & 0.041 & 0.681 & 0.809 \\
\hline
\end{tabular}

$$
\operatorname{RMSD}=\sqrt{\frac{\sum_{\omega=1}^{\Omega}\left(\hat{x}_{\omega}-x_{\omega}\right)^{2}}{\Omega}},
$$

with $\hat{x}_{\omega}$ an observation in a set of $\Omega$ observations (i.e., a value predicted by the model), the associated benchmark value in the test set of which is $x_{\omega}$.

In order to train the RBFNs, historical data are normalized to zero mean and unit variance and subsequently clustered using the $k$-means algorithm, with the number of clusters equal to the number of hidden nodes (Nabney, 2002). Using the expectation maximization algorithm (Dempster, Laird, \& Rubin, 1977), Gaussians are fit by optimizing the centers (initialized as the cluster centers). Gaussian widths equal the maximum squared Euclidian distance between the function centers. Finally, internal weights of the RBFNs are optimized, such that the sum of squared errors is minimized. The results in Table 1 indicate that in general, parameter estimation based on real-time available data leaves some room for improvement, albeit to a lesser extent for $\alpha_{k}$. Nevertheless, the proposed online feedback mechanism should (partially) compensate for this.

\section{PERFORMANCE EVALUATION}

The performance of the DynER system is assessed in real TAC SCM games, as specified by Collins et al. (2005). The random settings of TAC SCM, like in customer demand, introduce a problem when using this testing environment to compare two different approaches. This randomness is partly controlled by using the TAC SCM server (Sodomka, Collins, \& Gini, 2007), in which random seeds used for generating market conditions can be re-used. However, the stochastic behavior of participating agents cannot be controlled, as this is not generated on the server. Research shows that under equal market conditions, such stochastic behavior does not have a significant impact on the agent profit levels and that profitability differences between agents can be reliably detected in approximately 40 games (Sodomka et al., 2007). In Ketter, Collins, and Gini (2010), the authors show how the various agents use more or less standard supply chain decision strategies in their designs.

We therefore evaluate the performance of the DynER model in 40-game experiments on a controlled server. These 40 games are used for validation purposes 
Table 2: Mean and standard deviation of final bank account balance (in millions) per agent, calculated over all experiments for both the benchmark and the DynER configuration. The performance difference $\Delta$ between both configurations and the associated statistical significance (sig.) are reported as well.

\begin{tabular}{|c|c|c|c|c|c|c|}
\hline \multirow[b]{2}{*}{ Agent } & \multicolumn{2}{|c|}{ Benchmark } & \multicolumn{2}{|c|}{ DynER } & \multicolumn{2}{|c|}{$\Delta$} \\
\hline & Mean & St dev & Mean & St dev & $\%$ & Sig. \\
\hline MT & -0.355 & 5.451 & 1.345 & 4.109 & 478.4 & 0.000 \\
\hline TT & 5.144 & 5.569 & 5.295 & 5.501 & 2.9 & 0.528 \\
\hline DM & 3.907 & 4.000 & 4.249 & 3.845 & 8.8 & 0.125 \\
\hline PA & 5.627 & 5.198 & 5.391 & 5.143 & -4.2 & 0.096 \\
\hline MC & -2.536 & 3.845 & -2.487 & 4.273 & 1.9 & 0.757 \\
\hline $\mathrm{CA}$ & -7.568 & 5.794 & -7.986 & 5.432 & -5.5 & 0.166 \\
\hline
\end{tabular}

and are not part of the training and test sets for our model. Each experiment set consists of a paired evaluation of the performance of the benchmark (Ketter et al., 2009, 2012) and the DynER system under equal market definitions. The configurations can be tested in a highly competitive setting, in which MinneTAC competes against world's leading TAC SCM trading agents: TacTex (Pardoe \& Stone, 2006b) (TT), DeepMaize (Kiekintveld, Miller, Jordan, \& Wellman, 2006; Kiekintveld, Miller, Jordan, Callender, \& Wellman, 2009) (DM), PhantAgent (Stan, Stan, \& Florea, 2006) (PA), the CrocodileAgent (Podobnik, Petric, \& Jezic, 2006) (CA), and Mertacor (Chatzidimitriou, Symeonidis, Kontogounis, \& Mitkas, 2008) (MC). TT predicts demand using a Bayesian approach and estimates offer acceptance probabilities using linear regression. It adapts these offer acceptance probability estimations to its opponents' behavior. DM uses a gradient descent algorithm to find offer prices that optimize the expected value of the resulting orders. PA and CA use simple heuristics for determining what to sell for what price. MC predicts the winning bid per RFQ using a regression model, complemented with a pricefollowing correction mechanism. The acceptance probabilities are subsequently estimated using a $k$-nearest neighbors algorithm (MacQueen, 1967).

Performance differences are assessed with respect to their statistical relevance using a paired, two-sided Wilcoxon signed-rank test (Gibbons, 1985; Hollander \& Wolfe, 1999), which evaluates the hypothesis that the differences between paired observations are symmetrically distributed around a median equal to zero. If this null hypothesis is rejected, at a significance level of 0.05 , the compared samples are labeled significantly different.

Tables 2 and 3 illustrate the observed performance of the benchmark and the DynER configurations of MinneTAC (MT), with respect to the performance of MinneTAC's competitors. The number of obtained orders obtained by MinneTAC decreases by about $3 \%$ when introducing adaptivity into the product pricing process, while MinneTAC's profits significantly increase by $478 \%$, thus improving its competitive position. The mean difference between the bank account balance of MinneTAC and its competitors significantly improves by $136 \%$ (in absolute terms) from -1.270 million (standard deviation 2.742 million) to 0.452 million 
Table 3: Mean and standard deviation of order volume (in thousands) per agent, calculated over all experiments for both the benchmark and the DynER configuration. The performance difference $\Delta$ between both configurations and the associated statistical significance (sig.) are reported as well.

\begin{tabular}{|c|c|c|c|c|c|c|}
\hline \multirow[b]{2}{*}{ Agent } & \multicolumn{2}{|c|}{ Benchmark } & \multicolumn{2}{|c|}{ DynER } & \multicolumn{2}{|c|}{$\Delta$} \\
\hline & Mean & St dev & Mean & St dev & $\%$ & Sig. \\
\hline MT & 4.131 & 0.569 & 4.016 & 0.370 & -2.8 & 0.040 \\
\hline TT & 6.646 & 0.485 & 6.510 & 0.533 & -2.0 & 0.000 \\
\hline DM & 5.279 & 0.589 & 5.424 & 0.606 & 2.8 & 0.000 \\
\hline PA & 6.007 & 0.489 & 6.021 & 0.499 & 0.2 & 0.323 \\
\hline MC & 6.493 & 0.404 & 6.525 & 0.425 & 0.5 & 0.175 \\
\hline $\mathrm{CA}$ & 5.617 & 0.540 & 5.579 & 0.581 & -0.7 & 0.605 \\
\hline
\end{tabular}

(standard deviation 1.803 million). Additionally, the difference between MinneTAC's score and the top score in a game significantly improves by $27 \%$ from -6.879 million (standard deviation 2.987 million) to -5.044 million (standard deviation 2.365 million). The decreased standard deviations suggest that the performance of MinneTAC is more stable over all experiments when utilizing the DynER approach instead of using the single-layered ER approach. As the DynER model has been designed to facilitate tactical product pricing, an intuitive explanation for MinneTAC's improved performance may be improvements in meeting target sales quotas. Later, we will discuss this in more detail.

Improvements in MinneTAC's bidding behavior can be observed in its bid efficiency. Bid efficiency is the revenue of a bid on an arbitrary RFQ, expressed in terms of the maximum revenue that could have been obtained from that RFQ, given its reservation price and competitor's offers (Jordan, Kiekintveld, Miller, \& Wellman, 2007). The optimal bid efficiency is equal to 1 . A bid efficiency less than 1 implies underpricing, whereas a bid efficiency greater than 1 implies overpricing. For the orders that MinneTAC does obtain, the mean bid efficiency increases slightly, yet significantly by $0.21 \%$ from 0.954 (standard deviation 0.005 ) to 0.956 (standard deviation 0.007) over all experiments. This implies that the prices of acquired orders tend to be closer to second lowest prices yielding a reduced margin between customers' reservation prices and realized order prices. In cases of overpricing, the mean bid efficiency significantly improves by $1.41 \%$ from 1.058 (standard deviation 0.028) to 1.043 (standard deviation 0.008), indicating that MinneTAC's lost bids are closer to the winning bid. The decreased standard deviation indicates a more stable performance of our acceptance probability estimation process in the case of non-winning offers. These observations suggest that DynER's acceptance probability estimations are better than those in the benchmark configuration. The order price distribution estimations (underlying acceptance probability estimations) in Figure 3 support our claim. Clearly, in the typical scenario depicted in Figure 3, the distribution of realized offer prices estimated by using DynER, given any of five considered dominant regimes, is closer to the real distribution than those estimated by the one-layered benchmark. 
Figure 3: Typical distribution of realized offer prices for a particular good on an arbitrary day, estimated by benchmark and DynER approaches for any of five dominant regimes.

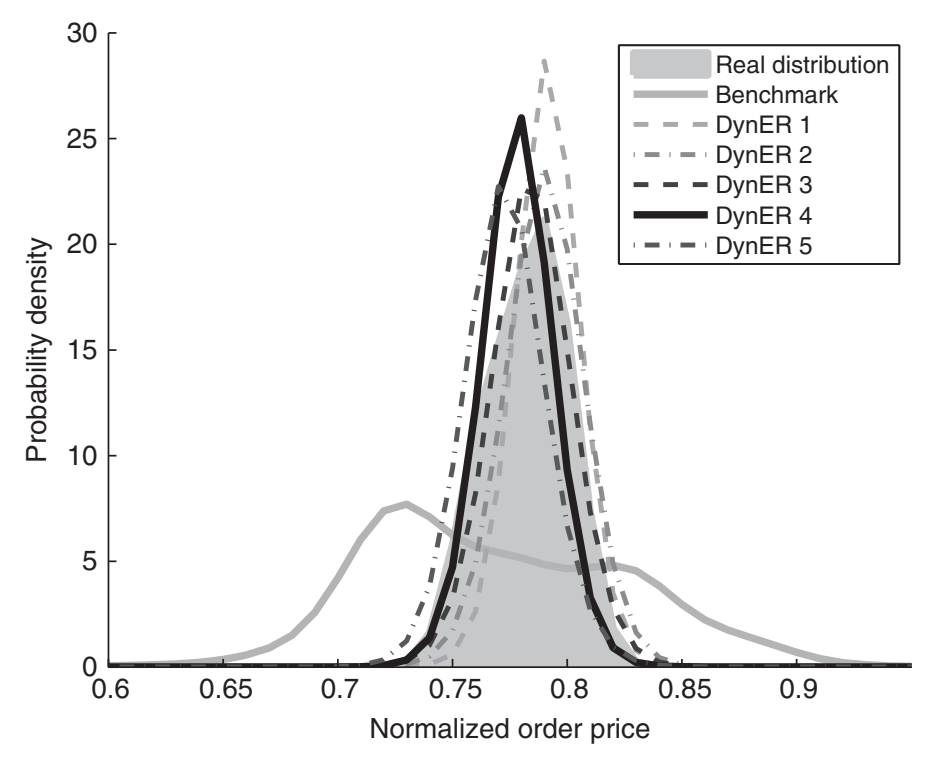

An intuitive explanation for the increase in profits could be that products are typically sold at higher prices when tactical sales pricing is introduced or the agent is able to sell significantly more products. However, neither of these seem to be the case with DynER. DynER results in a 3\% decrease in the number of orders obtained and the average price of products sold decreases by $2 \%$. Therefore, the increase in profits can be entirely attributed to reduction in costs. The cost has multiple components, namely, production cost as well as components and finished good inventory costs. Further analysis of our experimental data shows that approximately $65 \%$ of the observed increase in profits can be attributed to total material costs, which have significantly decreased by about $5 \%$. This may be the result of a minor decrease in the need for components due to the slightly decreased number of obtained orders. The remaining $35 \%$ of the observed increase in profits can be attributed to a significant decrease of both storage costs and interest by $13 \%$. Therefore, the proposed adaptive tactical product pricing approach results in higher profits by bidding better matched prices to achieve target sales quota to optimize its inventory levels, thus decreasing storage costs as well as interest paid.

Interestingly, even though the procurement strategy is identical in both configurations, we see significant differences in component inventory costs. Therefore, it is useful to examine this phenomenon in more detail. Total inventory costs are proportional to the number of stocked items and the time these items are in stock. Hence, an intuitive explanation for the significant drop in inventory costs when introducing adaptivity would be a drop in inventory levels. Indeed, inventory levels are significantly reduced by approximately $7 \%$ on average. This suggests that less 
Figure 4: Typical sales and inventory levels of finished goods and components over time.

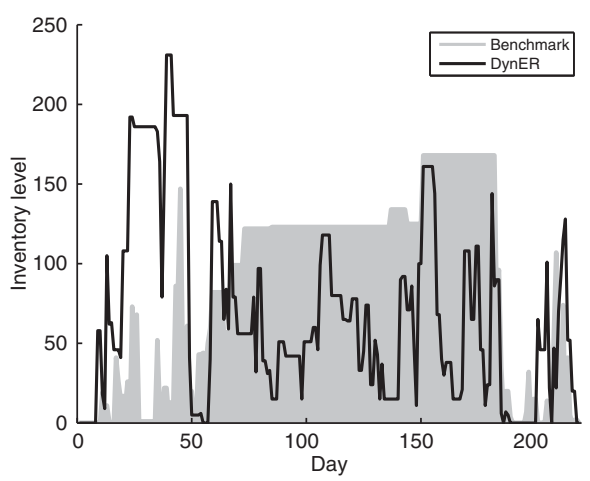

(a) Inventory levels of a typical product.

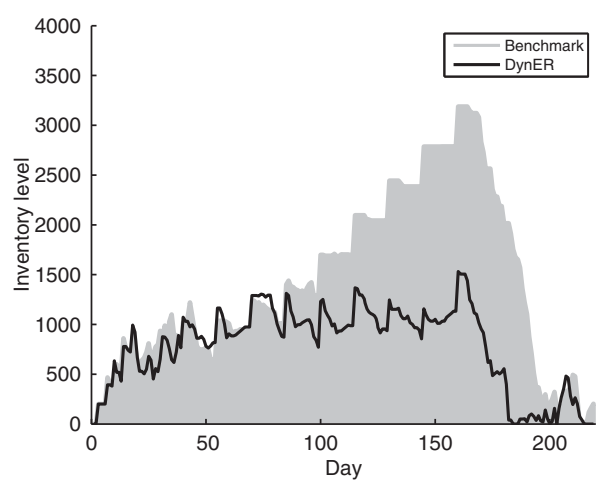

(b) Inventory levels of a typical component.

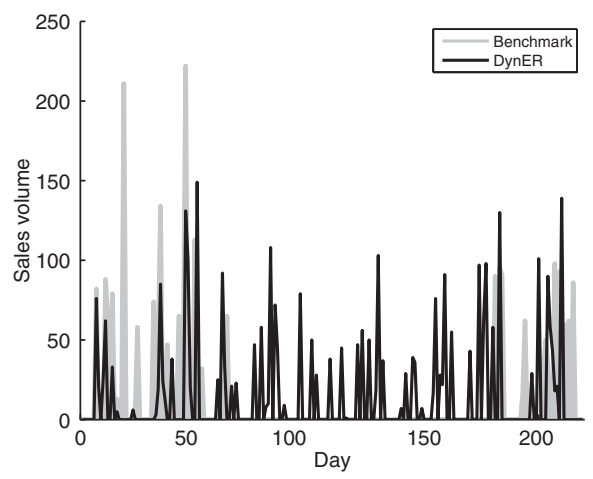

(c) Sales volume of a typical product.

inventory is built up due to more efficient pricing. Figure 4(a) shows the finished goods inventory levels, while the average inventory levels are not significantly different in the two configurations, in the single-layered model inventories are typically held for a much longer time period resulting in higher inventory costs. As Figure 4(b) indicates, the component inventory levels are significantly lower (by $25 \%$ ) with DynER. This reflects selling the right product at the right time to use the production facility more efficiently. DynER's consistent sales strategy is clearly reflected in Figure 4 as opposed to single-layered model where sales are concentrated at the beginning and the end of the sales cycle.

More detailed examination of MinneTAC's inventory levels reveals that, on average, the mean number of consecutive days that MinneTAC's inventory levels remain unchanged decreases significantly by $16 \%$. For MinneTAC's component inventories, the decrease is approximately $6 \%$, whereas for finished goods inventories, the decrease is about $22 \%$. The mean number of consecutive days that MinneTAC's inventories do not decrease due to a lack of successful sales attempts significantly decreases by $15 \%$ on average, where finished goods inventories and 
Figure 5: Typical overall MinneTAC market shares and unmet demand levels over time.

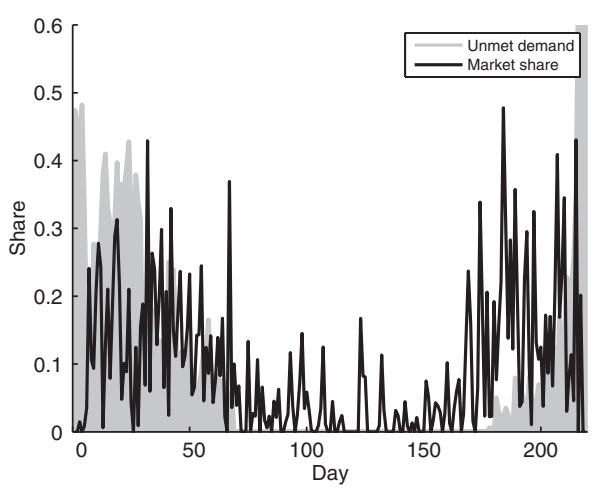

(a) Benchmark.

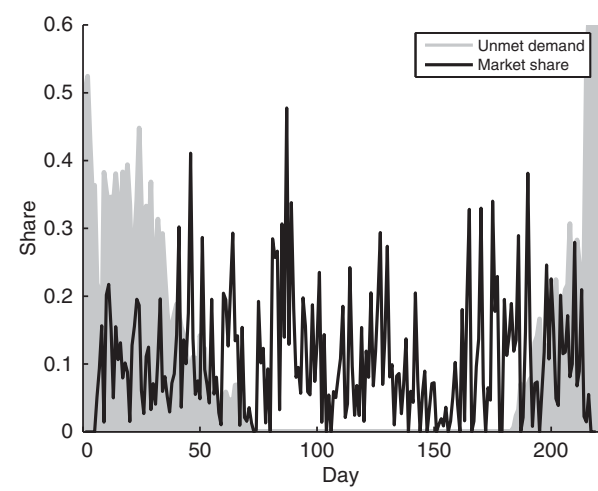

(b) DynER.

component inventories, respectively, account for an approximate $18 \%$ and $12 \%$ decrease in time in between inventory decreases. Moreover, the mean number of consecutive days without an increase of inventory levels decreases by about $7 \%$. This significant decrease is about $10 \%$ for finished goods inventories and an insignificant $2 \%$ decrease for component inventory levels.

To summarize, the introduction of tactical consideration in product pricing causes inventories to move faster, at a more steady pace. DynER causes MinneTAC to sell more often, resulting in a faster decrease of finished goods inventories and, to replenish these finished goods inventories, the corresponding constituent components are used in production thereby decreasing component inventories. The frequency of replenishment in component inventories does not increase significantly, as the employed procurement strategy — which already provides for very frequent procurement-is not changed in this experimental setup. The standard deviation of MinneTAC's sales quantities may be a more direct indicator of the change in sales behavior. This standard deviation on average decreases by $36 \%$ when DynER is used, which suggests that MinneTAC's sales performance is more stable over time.

The tactical change of the MinneTAC agent introduced by the DynER approach has its effects on MinneTAC's market position as well as on the supply chain as a whole, as illustrated in Figure 5. First, the number of days when all customer demand is met by the participating agents, on average, increases by $6 \%$. While this is beneficial to customers, it introduces higher competition for participating agents for a longer duration. Interestingly, it is in these highly competitive periods of time, when there is no excess demand, that our tactically focused strategy significantly improves MinneTAC's market share (by 66\%). In contrast, MinneTAC's market share in less competitive phases of the game is hardly affected. The dynamic tactical product pricing mechanism allows us to better adjust our prices during wellbalanced regimes, where demand can be satisfied with existing supply. This enables us to keep inventories balanced and to realize a lean, cost-effective sales process. 


\section{CONCLUSIONS AND FUTURE WORK}

When product pricing strategies are linked to price distribution estimates and real-time information is taken into account, the relation between data and price distribution parameters can be dynamically modeled using ERs (characterizing market conditions) and correction terms (accounting for market feedback). In a constrained environment like TAC SCM, ER estimates contribute to profit maximization when they are used to differentiate product pricing strategies. Test results indicate that in highly competitive settings, this novel innovative tactical approach, comprising of a two-layered machine technique, significantly improves the performance over a single-layered strategic product pricing approach.

The tactical adaptive product pricing mechanism performs better in estimating the high and low ends of price distributions (bid efficiency increases), thus better tracking the price distributions in the price range over a short period of time. Conversely, these distributions do not capture the full details of the distribution, not taking advantage of long-term market trends. Therefore, we do not see an increase in either sales price nor sales volume. However, profits increase significantly due to better alignment of sales quotas and finished goods inventories, which overall reduces inventory holding costs.

While the performance of our model significantly improves compared to using only ERs to predict prices, some aspects warrant future research. We are currently working on an ensemble prediction method, where we use the onelayered regime model for strategic decision making, and the two-layered regime model for tactical decision making. Since the methods might work well for different planning horizons, we plan to perform ensemble prediction for all days over the planning horizon. This should result in an overall better sales performance.

\section{References}

Adida, E., \& Perakis, G. (2010). Dynamic pricing and inventory control: Uncertainty and competition. Operations Research, 58(2), 289-302.

Araman, V., \& Caldentey, R. (2009). Dynamic pricing for nonperishable products with demand learning. Operations Research, 57(5), 1169-1188.

Besbes, O., \& Zeevi, A. (2009). Dynamic pricing without knowing the demand function: Risk bounds and near-optimal algorithms. Operations Research, 57(6), 1407-1420.

Bichler, M., Gupta, A., \& Ketter, W. (2010). Designing smart markets. Information Systems Research, 21(4), 688-699.

Chatzidimitriou, K., Symeonidis, A., Kontogounis, I., \& Mitkas, P. (2008). Agent Mertacor: A robust design for dealing with uncertainty and variation in SCM environments. Expert Systems with Applications, 35(3), 591-603.

Chopra, S., \& Meindl, P. (2004). Supply chain management. New Jersey, USA: Pearson Prentice Hall.

Coleman, T., \& Li, Y. (1996). An interior trust region approach for nonlinear minimization subject to bounds. SIAM Journal on Optimization, 6(2), 418445 . 
Collins, J., Arunachalam, R., Sadeh, N., Eriksson, J., Finne, N., \& Janson, S. (2005). The supply chain management game for the 2006 trading agent competition. Technical Report CMU-ISRI-05-132, Pittsburgh, PA: Carnegie Mellon University.

Collins, J., Ketter, W., \& Gini, M. (2009). Flexible decision control in an autonomous trading agent. Electronic Commerce Research and Applications, $8(2), 91-105$.

Collins, J., Ketter, W., \& Gini, M. (2010a). Flexible decision support in dynamic interorganizational networks. European Journal of Information Systems, 19(3), 436-448.

Collins, J., Ketter, W., \& Sadeh, N. (2010b). Pushing the limits of rational agents: The trading agent competition for supply chain management. AI Magazine, $31,63-80$.

Dasgupta, P., \& Hashimoto, Y. (2004). Multi-attribute dynamic pricing for online markets using intelligent agents. Proceedings of the 3rd International Joint Conference on Autonomous Agents and Multiagent Systems (AAMAS 2004). New York, NY: IEEE Computer Society, 277-284.

Dempster, A., Laird, N., \& Rubin, D. (1977). Maximum likelihood from incomplete data via the EM algorithm. Journal of the Royal Statistical Society B, 39(1), 1-38.

Gibbons, J. (1985). Nonparametric statistical inference (2nd Edition). New York City, NY, USA: McGraw-Hill.

Groves, W., Collins, J., Gini, M., \& Ketter, W. (2014). Agent-assisted supply chain management: Analysis and lessons learned. Decision Support Systems, 57, 274-284.

Hathaway, R., \& Bezdek, J. (1993). Switching regression models and fuzzy clustering. IEEE Transactions on Fuzzy Systems, 1(3), 195-204.

Hollander, M., \& Wolfe, D. (1999). Nonparametric statistical methods (2nd Edition). New York City, NY, USA: John Wiley \& Sons.

Jelinek, M., \& Bergey, P. (2013). Innovation as the strategic driver of sustainability: Big data knowledge for profit and survival. IEEE Engineering Management Review, 41(2), 14-22.

Jones, M. (1989). Discretized and interpolated kernel density estimates. Journal of the American Statistical Association, 84(407), 733-741.

Jordan, P., Kiekintveld, C., Miller, J., \& Wellman, M. (2007). Market efficiency, sales competition, and the bullwhip effect in the TAC SCM tournaments. Lecture Notes in Computer Science, 4452, 62-74.

Kapadia, A., Chan, W., \& Moye, L. (2005). Mathematical statistics with applications. Boca Raton, FL, USA: CRC Press.

Ketter, W., Collins, J., \& Gini, M. (2010). Coordinating decisions in a supplychain trading agent. In W. Ketter, H. L. Poutre, N. M. Sadeh, O. Shehory, \& W. Walsh (Eds.), Agent-mediated electronic commerce $X$ and trading agent design and analysis VI, vol. 44 of Lecture Notes in Business Information Processing. New York City, NY, USA: Springer, 161-174. 
Ketter, W., Collins, J., Gini, M., Gupta, A., \& Schrater, P. (2009). Detecting and forecasting economic regimes in multi-agent automated exchanges. Decision Support Systems, 47(4), 307-318.

Ketter, W., Collins, J., Gini, M., Gupta, A., \& Schrater, P. (2012). Real-time tactical and strategic sales management for intelligent agents guided by economic regimes. Information Systems Research, 23, 1263-1283.

Ketter, W., Collins, J., \& Reddy, P. (2013a). Power TAC: A competitive economic simulation of the smart grid. Energy Economics, 39, 262-270.

Ketter, W., Peters, M., \& Collins, J. (2013b). Autonomous agents in future energy markets: The 2012 Power Trading Agent Competition. Association for the Advancement of Artificial Intelligence (AAAI) Conference Proceedings. Bellevue, Washington.

Ketter, W., \& Symeonidis, A. (2012). Competitive benchmarking: Lessons learned from the Trading Agent Competition. AI Magazine, 33(1), 103-107.

Kiekintveld, C., Miller, J., Jordan, P., Callender, L., \& Wellman, M. (2009). Forecasting market prices in a supply chain game. Electronic Commerce Research and Applications, 8(2), 63-77.

Kiekintveld, C., Miller, J., Jordan, P., \& Wellman, M. (2006). Controlling a supply chain agent using value-based decomposition. Proceedings of the 7th ACM Conference on Electronic Commerce (EC'06). New York, NY: ACM, 208217.

Kovalchuk, Y., \& Fasli, M. (2008). Adaptive strategies for predicting bidding prices in supply chain management. Proceedings of the 10th International Conference on Electronic Commerce (ICEC 2008). Innsbruck, Austria.

Li, C., Giampapa, J., \& Sycara, K. (2004). Bilateral negotiation decisions with uncertain dynamic outside options. Proceedings of the First IEEE International Workshop on Electronic Contracting. Washington, DC: IEEE Computer Society, 54-61.

MacQueen, J. (1967). Some methods of classification and analysis of multivariate observations. Proceedings of the 5th Berkeley Symposium on Mathematical Statistics and Probability. 281-297.

Marchette, D., \& Priebe, C. (1990). The adaptive kernel neural network. Mathematical and Computer Modelling, 14, 328-333.

Massey, C., \& Wu, G. (2005). Detecting regime shifts: The causes of under- and overreaction. Management Science, 51(6), 932-947.

Mitchell, T. (1997). Machine learning. McGraw-Hill Series in Computer Science. New York City, NY, USA: McGraw-Hill.

Mood, A., Graybill, F., \& Boes, D. (1974). Introduction to the theory of statistics. New York City, NY, USA: McGraw-Hill.

Mount, T., Ning, Y., \& Cai, X. (2006). Predicting price spikes in electricity markets using a regime-switching model with time-varying parameters. Energy Economics, 28(1), 62-80.

Nabney, I. (2002). NETLAB: Algorithms for pattern recognition. New York City, NY, USA: Springer. 
Nair, A., Narasimhan, R., \& Choi, T. (2009). Supply networks as a complex adaptive system: Toward simulation-based theory building on evolutionary decision making. Decision Sciences, 40(4), 783-815.

Pardoe, D., \& Stone, P. (2006a). Predictive planning for supply chain management. Proceedings of the 16th International Conference on Automated Planning and Scheduling. 21-30.

Pardoe, D., \& Stone, P. (2006b). TacTex-05: A champion supply chain management agent. Proceedings of the 21 st National Conference on Artificial Intelligence. 1489-94.

Pathak, S., Day, J., Nair, A., Sawaya, W., \& Kristal, M. (2007). Complexity and adaptivity in supply networks: Building supply network theory using a complex adaptive systems perspective. Decision Sciences, 38(4), 547-580.

Podobnik, V., Petric, A., \& Jezic, G. (2006). The CrocodileAgent: Research for efficient agent-based cross-enterprise processes, vol. 4277 of Notes in Computer Science. New York City, NY, USA: Springer, 752-762.

Saha, S., Biswas, A., \& Sen, S. (2005). Modeling opponent decision in repeated one-shot negotiations. Proceedings of the 4th International Joint Conference on Autonomous Agents and Multiagent Systems. New York, NY: ACM, 397403.

Sodomka, E., Collins, J., \& Gini, M. (2007). Efficient statistical methods for evaluating trading agent performance. Proceedings of the 22nd AAAI Conference on Artificial Intelligence. Vancouver, British Columbia, Canada: AAAI Press, 770-775.

Sohn, S., Moon, T., \& Seok, K. (2009). Optimal pricing for mobile manufacturers in competitive market using genetic algorithm. Expert Systems with Applications, 36(2), 3448-3453.

Sprague, R., \& Carlson, E. (1982). Buidling effective decision support systems. New Jersey, USA: Prentice Hall.

Stan, M., Stan, B., \& Florea, A. (2006). A dynamic strategy agent for supply chain management. Proceedings of the 8th International Symposium on Symbolic and Numeric Algorithms for Scientific Computing. Washington, DC: IEEE Computer Society, 227-232.

Swaminathan, J., Smith, S., \& Sadeh, N. (1998). Modeling supply chain dynamics: A multiagent approach. Decision Sciences, 29(3), 607-632.

Swaminathan, J., \& Tayur, S. (2003). Models for supply chains in e-business. Management Science, 49(10), 1387-1406.

Swedish Institute for Computer Science (2004-2012). Trading agent competition. Website, accessed March 2013, Available online, http://www.sics.se/tac.

Tang, C., \& Yin, R. (2007). Responsive pricing under supply uncertainty. European Journal of Operational Research, 182(1), 239-255.

Titterington, D., Smith, A., \& Makov, U. (1985). Statistical analysis of finite mixture distributions. New York, NY: John Wiley and Sons. 
University of Minnesota (2003-2012). Trading agent competition. Website, accessed March 2013, Available online, http://tac.cs.umn.edu/.

van den Berg, J., Kaymak, U., \& van den Bergh, W. (2004). Financial markets analysis by using a probabilistic fuzzy modelling approach. International Journal of Approximate Reasoning, 35(3), 291-305.

Van der Zee, D., \& van der Vorst, J. (2005). A modeling framework for supply chain simulation: Opportunities for improved decision making. Decision Sciences, 36(1), 65-95.

van Ryzin, G., \& Vulcano, G. (2004). Optimal auctioning and ordering in an infinite horizon inventory-pricing system. Operations Research, 52(3), 346-367.

Walsh, W., Parkes, D., Sandholm, T., \& Boutilier, C. (2008). Computing reserve prices and identifying the value distribution in real-world auctions with market disruptions. Proceedings of the 23rd AAAI Conference on Artificial Intelligence. Chicago, IL, 1499-1502.

\section{APPENDIX}

A summary of the mathematical notation used in this article can be found in Table A1.

Table A1: Summary of notation.

\begin{tabular}{|c|c|}
\hline Symbol & Definition \\
\hline$\alpha$ & $\begin{array}{l}\text { Median offer price representation in the truncated log-logistic } \\
\text { distribution }\end{array}$ \\
\hline$\beta$ & Smoothing factor for exponential smoothing \\
\hline$\gamma$ & $\begin{array}{l}\text { Quantification of the tightness of the truncated log-logistic } \\
\text { distribution }\end{array}$ \\
\hline$\epsilon$ & $\begin{array}{l}\text { Correction term accounting for the ratio of received to predicted } \\
\text { orders }\end{array}$ \\
\hline$\tilde{\epsilon}$ & $\begin{array}{l}\text { Smoothed correction term accounting for the ratio of received to } \\
\text { predicted orders }\end{array}$ \\
\hline$\sigma_{h}^{2}$ & Variance of hidden unit $h$ \\
\hline$d\left(\vec{x}_{h}, \vec{x}\right)$ & $\begin{array}{l}\text { Euclidean distance between local function center } \vec{x}_{h} \text { and a vector of } \\
\quad \text { features } \vec{x} \text { for hidden unit } h\end{array}$ \\
\hline$f(p ; \theta)$ & $\begin{array}{l}\text { Distribution of normalized valid offer price } p \text {, with } \theta \text { a vector of } \\
\text { parameters }\end{array}$ \\
\hline$F(p ; \theta)$ & $\begin{array}{l}\text { Cumulative distribution of normalized valid offer price } p \text {, with } \theta \text { a } \\
\text { vector of parameters }\end{array}$ \\
\hline$F_{1}(p ; \theta)$ & $\begin{array}{l}\text { Cumulative distribution of minimum normalized valid offer price } p \text {, } \\
\text { with } \theta \text { a vector of parameters }\end{array}$ \\
\hline$h(\vec{x})$ & $\begin{array}{l}\text { Relation between a vector of real-time available information } \vec{x} \text { and a } \\
\text { value to be estimated (e.g., distribution parameter) }\end{array}$ \\
\hline$\hat{h}(\vec{x})$ & $\begin{array}{l}\text { Approximated relation between a vector of real-time available } \\
\text { information } \vec{x} \text { and a value to be estimated (e.g., distribution } \\
\text { parameter) }\end{array}$ \\
\hline
\end{tabular}


Table A1: Continued

\begin{tabular}{ll}
\hline Symbol & \\
\hline$H$ & Number of hidden units \\
$K_{h}\left(d\left(\vec{x}_{h}, \vec{x}\right)\right)$ & $\begin{array}{l}\text { Kernel function of hidden unit } h \text {, depending on the distance between } \\
\text { the local function center } \vec{x}_{h} \text { and a vector of features } \vec{x}\end{array}$ \\
$L(\theta ; p)$ & $\begin{array}{l}\text { Negated log-likelihood function of parameters in vector } \theta \text { for a } \\
\text { sample of normalized valid offer prices } p\end{array}$ \\
$m$ & Number of RFQs issued \\
$M$ & Number of economic regimes \\
$n$ & Number of normalized valid offer prices \\
$n p$ & Normalized order price \\
$\widetilde{n p}$ & Smoothed normalized mid-range order price realized over all RFQs \\
& of a product on a game day \\
$p$ & Normalized valid offer price \\
$p^{*}$ & Optimal normalized valid offer price, expected to yield sales quota \\
$p^{*^{\prime}}$ & Corrected optimal normalized valid offer price, expected to yield \\
$P(o \mid p)$ & sales quota \\
$P(o \mid p)^{\prime}$ & Probability that a customer will place an order $o$ with an agent, given \\
$P\left(R_{k}\right)$ & its normalized valid offer price $p$ \\
$q$ & Corrected probability that a customer will place an order $o$ with an \\
$q^{*}$ & agent, given its normalized valid offer price $p$ \\
$q^{*^{\prime}}$ & Probability of regime $R_{k}$ \\
$u$ & Proportion of actually received orders \\
$w$ & Sales quota (desired obtained proportion of orders) \\
$x$ & Corrected sales quota (desired obtained proportion of orders) \\
$\hat{x}$ & Upper bound of the double-bounded log-logistic distribution \\
\hline & Weight of an activation unit
\end{tabular}

